\title{
Big and small numbers: Empirical support for a single, flexible mechanism for numerosity perception
}

\author{
Rakesh Sengupta $^{1,2}$. S. Bapiraju ${ }^{3}$. David Melcher ${ }^{4}$
}

Published online: 28 October 2016

(C) The Psychonomic Society, Inc. 2016

\begin{abstract}
The existence of perceptually distinct numerosity ranges has been proposed for small (i.e., subitizing range) and larger numbers based on differences in precision, Weber fractions, and reaction times. This raises the question of whether such dissociations reflect distinct mechanisms operating across the two numerosity ranges. In the present work, we explore the predictions of a single-layer recurrent on-center, off-surround network model of attentional priority that has been applied to object individuation and enumeration. Activity from the network can be used to model various phenomena in the domain of visual number perception based on a single parameter: the strength of inhibition between nodes. Specifically, higher inhibition allows for precise representation of small numerosities, while low inhibition is preferred for high numerosities. The model makes novel predictions, including that enumeration
\end{abstract}

Rakesh Sengupta

rakesh@cse.yorku.ca

S. Bapiraju

raju.bapi@iiit.ac.in

David Melcher

david.melcher@unitn.it

1 Department of Electrical Engineering and Computer Science, Lassonde Building, York University, 4700, Keele Street, Toronto, Ontario, M3J 1P3 Canada

2 Center for Cognitive Science, B. V. Raju Institute of Technology, Narsapur, Andhra Pradesh, 502313, India

3 Cognitive Science Lab, International Institute of Information Technology, Hyderabad, India

4 Center for Mind/Brain Sciences, University of Trento, Trento, Italy of small numerosities following large numerosities should result in longer reaction times than when a small numerosity trial following small numerosities. Moreover, the model predicts underestimation of number when a display containing a large number of items follows a trial with small numerosities. We behaviorally confirmed these predictions in a series of experiments. This pattern of results is consistent with a single, flexible object individuation system, which can be modeled successfully by dynamic on-center, off-surround network model of the attentional priority (saliency) map.

Keywords Theoretical and computational models . Reaction time methods · Spatial vision

\section{Introduction}

Numerical cognition is grounded in the perceptual capacity to individuate or estimate the number of objects in a set (Halberda \& Feigenson, 2008; Piazza, Fumarola, Chinello, \& Melcher, 2011; Piazza, Pinel, Le Bihan, \& Dehaene, 2007). Enumeration of multiple visual objects gives rise to two behaviorally distinct phenomena-subitizing and estimation-depending on the numerosity of the objects presented on a display (Kaufman, Lord, Reese, \& Volkmann, 1949). A rapid, precise, and confident enumeration strategy called subitizing works for smaller numerosity (generally less than 5), while a less accurate and slower enumeration strategy of estimation seems to operate at higher ranges of numerosity. The distinction between the ranges has been shown in Weber fractions (Burr, Turi, \& Anobile, 2010; Choo \& Franconeri, 2014) and precision measures (Whalen, Gallistel, \& Gelman, 1999).

It has been of considerable interest to understand the possible mechanisms for enumeration (Trick \& Pylyshyn, 
1994). Some have proposed that the subitizing range might reflect capacity limits in visuo-spatial object individuation (Melcher \& Piazza, 2011; Piazza et al., 2011), while estimation has been suggested to reflect coarse ensemble statistics measures (Ariely, 2001; Burr \& Ross, 2008; Feigenson, Dehaene, \& Spelke, 2004). Alternatively, it has been suggested that subitizing and estimation reflect a single mechanism operating at different numerical resolutions (Gallistel \& Gelman, 1992; Revkin, Piazza, Izard, Cohen, \& Dehaene 2008). However, recent studies have shown that precision for the two ranges do not scale evenly (Revkin et al., 2008). This has led some to suggest the possibility of separate mechanisms for the two enumeration strategies (Burr et al., 2010).

A recent study by Burr et al. (2010) suggests that subitizing and estimation share common pre-attentive mechanisms, but that subitizing makes use of additional attentional resources and thus is subject to capacity limits. One hypothesis is that subitizing reflects the activity of attentional priority (saliency) maps that represent at most a few salient objects (Knops, Piazza, Sengupta, Eger, \& Melcher, 2014; Melcher \& Piazza, 2011; Piazza et al., 2011). By definition, a saliency map does not represent all of the items in a large display, rather only the most relevant (and attended) objects. Thus, the link between subitizing and attention could be mediated by such a mechanism. There are several advantages to a priority/saliency map model explanation for capacity limits (for reviews, see Melcher and Piazza (2011); Franconeri, Alvarez, \& Cavanagh, 2013), including the fact that there is extensive neurophysiological and neuroimaging data supporting the existence of such maps (for reviews, see: Gottlieb and Goldberg (1999); Knops et al. (2014)) and that capacity limits emerge naturally out of such maps (Franconeri et al., 2013; Melcher \& Piazza, 2011).

A number of studies have indicated posterior parietal cortex as the locus of limits in object individuation, working memory, and enumeration (for a review, see: Piazza et al. (2007)). It has been suggested that numerosity detectors in the lateral intraparietal (LIP) region might be possible candidates for our perception of numbers (Piazza, Izard, Pinel, Le Bihan, \& Dehaene, 2004; Roggeman, Fias, \& Verguts 2010; Roitman, Brannon, \& Platt, 2007). These detectors might develop through self-organized or reinforcement learning during development (Dehaene \& Changeux, 1993; Grossberg \& Repin, 2003; Stoianov \& Zorzi, 2012). It is interesting to note that the LIP area is shared between visual spatial attention and visual short-term memory networks (Awh \& Jonides, 2001). Depending on the task (working memory task or enumeration) different fMRI activation patterns as a function of the number of items presented can be found in posterior parietal cortex (Kawasaki, Watanabe, Okuda, Sakagami, \& Aihara, 2008; Knops et al., 2014; Todd \& Marois, 2004, 2005).
In order to investigate the possible mechanisms underlying rapid enumeration, we previously modeled a recurrent on-center off-surround (OCOS) network that receives a normalized input based on locations occupied in the visual field and outputs a steady-state mean activation (Sengupta, Surampudi, \& Melcher, 2014). The normalization may take place through some method like divisive normalization as suggested by Reynolds and Heeger (2009). The model successfully captured a wide range of behavioral phenomena observed in enumeration research, including the distance effect (precision for numerosity discrimination for a given numerical distance is better for smaller numerosities than higher), Fechner's law (discrimination performance degrades for higher numerosities), as well as human numerosity comparison data collected by Piazza et al. (2004). In our model, subitization and estimation ranges emerge naturally with parametric variation of the recurrent inhibition strength of the network (see also Roggeman et al. (2010) for a similar approach). Moreover, the model also predicted that reaction times for enumeration would show hysteresis if the subjects were forced to change enumeration strategy by making them enumerate large numbers following several trials of small numerosity and vice versa. This novel prediction (explained in more detail in the next section) hinges on the idea that enumeration and estimation might be carried out by the same flexible network acting under a different level of inhibition between nodes. In the current work, we describe the model and its predictions, then present the experimental evidence for the model's predictions.

\section{Model}

\section{Description}

We have described the model in great detail in our previous work Sengupta et al. (2014). For the current purposes, we will draw attention to the salient features of the model behavior.

The model uses a single layer of $N$ completely connected self-excitatory nodes that laterally inhibit each other. Thus, here every node inhibits every other node, i.e., it is a fully connected network with all nodes as neighbors at unit distance. The strengths of self-excitation and lateral inhibition are given by $\alpha$ and $\beta$, respectively. The temporal dynamics of the system is given by the following equation,

$\dot{x}_{i}=\frac{d x_{i}}{d t}=-x_{i}+\alpha F\left(x_{i}\right)-\beta \sum_{j=1, j \neq i}^{N} F\left(x_{j}\right)+I_{i}+$ noise 
Table 1 Simulation parameters used in the computational model of object individuation and numerosity judgments

\begin{tabular}{ll}
\hline Parameter & Value \\
\hline$N$ & 70 \\
$\alpha$ & 2.2 \\
$\beta$ range & $0.01-0.15$ \\
Set size range & $1-64$ \\
Duration of stimulus presentation & 5 \\
(No. of time steps) & \\
Total duration of simulation in time steps & 50 \\
Number of simulations run & 100 \\
to compute the average & \\
\hline
\end{tabular}

where $x_{i}$ is the activation of $i$-th node. $I_{i}$ represents the transient input to the $i$-th node that lasts for finite time and has constant value in the interval $[0,1]$ in the duration. The node receives an input only if there is stimulus at the location the location represented by the particular node and zero otherwise. ${ }^{1} F(x)$ is the activation function given by

$F(x)= \begin{cases}0 & \text { for } x \leq 0 \\ \frac{x}{1+x} & \text { for } x>0\end{cases}$

For simulation purposes, we used the discrete form of Eq. 1. The updating of activation at time step $t$ is given by

$x_{i}(t)=\alpha F\left(x_{i}(t-1)\right)-\beta \sum_{j=1, j \neq i}^{N} F\left(x_{j}(t-1)\right)+I_{i}+$ noise

where the input was a step function that lasted for 5 time steps and then became 0 . The whole simulation was run for a total of 50 time steps. In line with our computational work (Sengupta et al., 2014), a network of 70 nodes was deployed (i.e., $N=70$ ). The relevant parameters are listed in Table 1 . The noise was sampled at each time step from a normal distribution with mean 0 and standard deviation of 0.03 .

\section{Model behavior}

The recurrent nature of the network, with its lateral inhibitory connection between nodes and self-excitations, creates a push-pull effect that drives the dynamics to a stable equilibrium. For instance, if the network has high inhibition, then only relatively few of the nodes may remain active at equilibrium, no matter how many nodes were excited initially. In the low-inhibition condition, however, even small perturbations created by noise can get amplified due to self-excitation in that particular node. Thus, even if a small number of nodes were excited initially in a

\footnotetext{
${ }^{1}$ In fact the input was clamped at 0.33 in line with Sengupta et al. (2014)
}

low-inhibition network, there might be a large number of active nodes in the final equilibrium state. The inhibition also controls the mean activation levels that can be reached in equilibrium. Figures 1 and 2 illustrate the activation patterns for different set sizes at different inhibition levels. As can be seen, higher inhibition acts like more focused (local) attention with capacity limits and lower inhibition acts like diffused (global) attention.

The choice of the network parameters was put into effect by rigorous convergence tests, detailed in Sengupta et al. (2014). Network behavior is robust over a range of simulation parameters and the results are not closely tied to a particular choice of parameters.

The output of the network is the mean steady-state activation achieved by the dynamics of the network at steadystate/equilibrium. The network mean activation is used as a surrogate for numerosity evaluation from the network. If numerosity judgments are to be made based on the mean activation of the network, then the mean activation should monotonically increase with numerosity (see Fig. 3). This constraint automatically gives us two regimes of numerosity in a self-organized manner-smaller numerosities are better enumerated at high $\beta$ and larger numerosities are better estimated at low $\beta$. As described above, the model can explain major behavioral performances in both subitizing and estimation range, such as the distance effect and numerosity comparison data for human adults (Sengupta et al., 2014).

\section{Predictions of the model}

In our earlier work (Sengupta et al., 2014), we derived an energy function in order to explore the stability of this kind of network. Reaction times (RT) were derived based on the assumption that RT correlates with the highest possible allowed fluctuation in energy. The expression for RT in the enumeration task is given as

$R T \sim \sum_{i} \int\left(1-\alpha\left(\frac{F\left(x_{i}\right)}{x_{i}}\right)^{2}\right) \dot{x}_{i}^{2} d t$

$\dot{x}_{i}=\frac{d x_{i}}{d t}$. See Appendix for a longer explanation for the choice of this particular expression.

The quantity on the right-hand side (see model description above for a definition of the terms) is proportional to the actual RT, but not equal to it and thus we refer to it as RT correlate or $R T_{\text {model }}$. Considering that high inhibition is better for enumerating smaller numerosities and low inhibition is better for accounting for large numerosities, we asked the question: what happens to RT when you enumerate smaller numerosity at low inhibition or you enumerate large numerosity at higher inhibition? In order to test the model predictions regarding such a switching cost, we took an average of RT correlates obtained through simulation for 

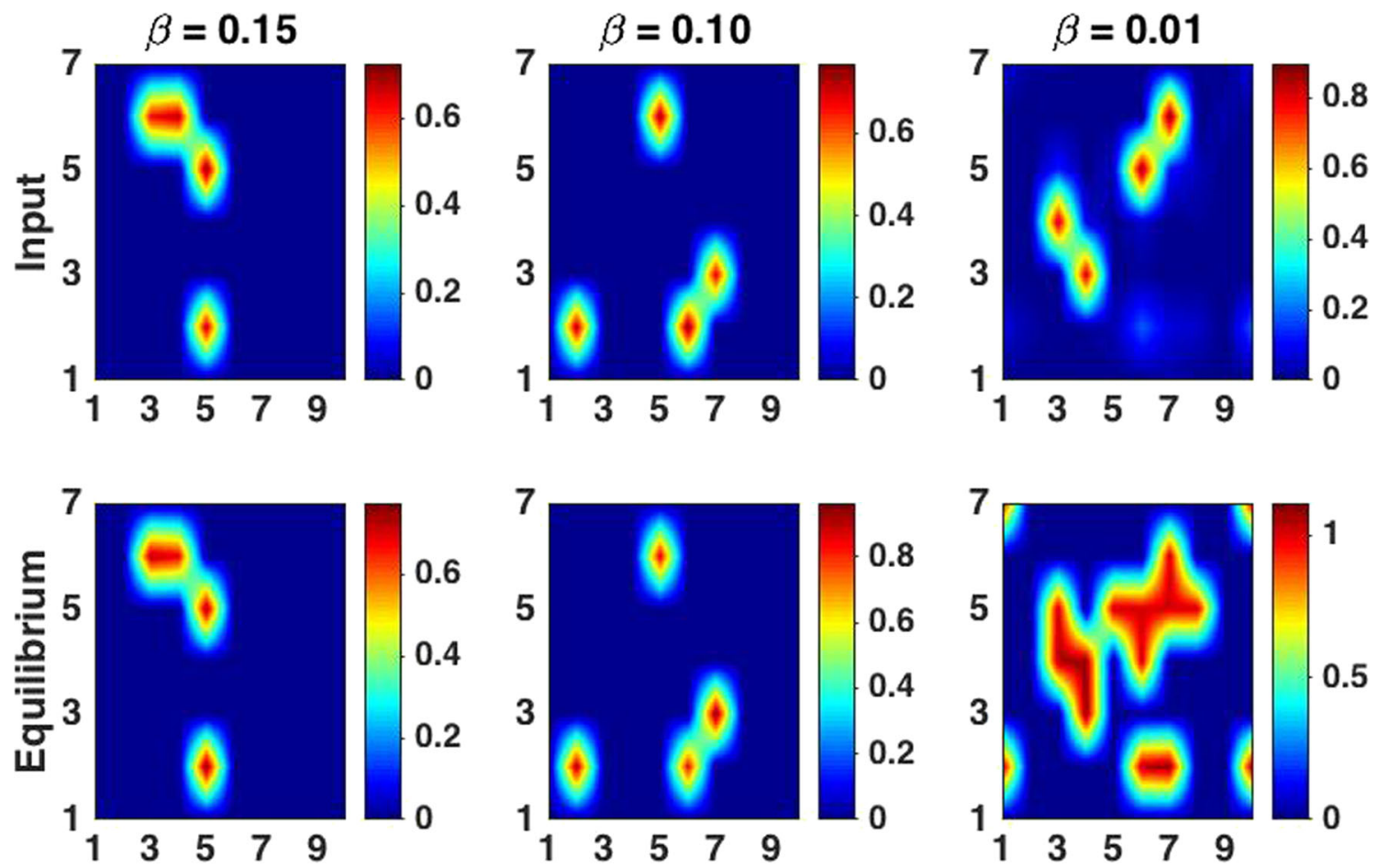

Fig. 1 Network is represented as a $10 \times 7$ rectangular grid with each point on the rectangle representing a node. All the nodes inhibit every other node and each node excites itself. Network dynamics for a small

higher inhibition ranges $(\beta=0.06-0.08)$ and lower inhibition ranges $(\beta=0.03-0.06){ }^{2}$ As shown in Fig. 4 , the model predicts a particular pattern of hysteresis. If one enumerates lower numerosity with low inhibition (a condition that might be manipulated experimentally by having a low numerosity enumeration task follow a series of high numerosity enumeration tasks) then the RTs should increase compared to standard RT for that range. In contrast, there is almost no RT cost for higher numerosity being enumerated at higher inhibition. Thus, we decided to test the prediction of the model with an enumeration task where a variable number of small numerosity enumeration (1-6 items) trials would follow a variable number of large numerosity estimation (24-34 items) trials and vice versa.

The model also gives us a measure of underestimation that would follow a numerosity comparison for higher numerosities. We can formulate a numerosity comparison

\footnotetext{
${ }^{2}$ The pattern of results is robust for range of parameter choices (see Appendix of Sengupta et al. (2014)). A similar pattern would be found if we varied the ranges keeping the difference of the means of the ranges of $\beta$ more than 0.02 with the low $\beta$ range being around 0.05 and lower. The particular choice of parameters was motivated by the experimental design, avoiding extreme ranges of inhibition but focusing instead on the middle region of $\beta$, i.e., $0.03-0.08$. To make the change between low and high inhibition regimes more plausible, we used this relatively small range of values for $\beta$ and simply divided it into low inhibition and higher inhibition.
}

number of inputs (4) at different levels of $\beta$. Top panels show the initial activations and bottom panels show final activation on a $10 \times 7$ grid representing 70 nodes $(N=70)$

measure from running a large number of simulations in order to check the probability of a particular numerosity being judged higher than a reference numerosity purely by comparing the mean activation values at steady state for different $\beta$. We can get the Weber fraction plots for this probability at different average $\beta$ values. The plots (see Fig. 5 clearly show that (i) the higher numerosities tend to be underestimated (the point of subjective equality or the $50 \%$ point corresponds to higher numerosity ratio), (ii) if we increase the average $\beta$, the point of subjective equality shifts right, pointing to higher underestimation. The exact underestimation values predicted for the numerosities used in the experimental results are shown in Fig. 6.

Thus, the model would predict greater underestimation at higher inhibition. This leads to the prediction that a high numerosity trial following low numerosity trials would suffer greater underestimation compared to a high numerosity trial following high numerosity trial.

\section{Methods}

\section{Participants}

A total of 21 adult volunteers participated in this study (age range, 24-32). Of this group, 12 were tested at the Center for 

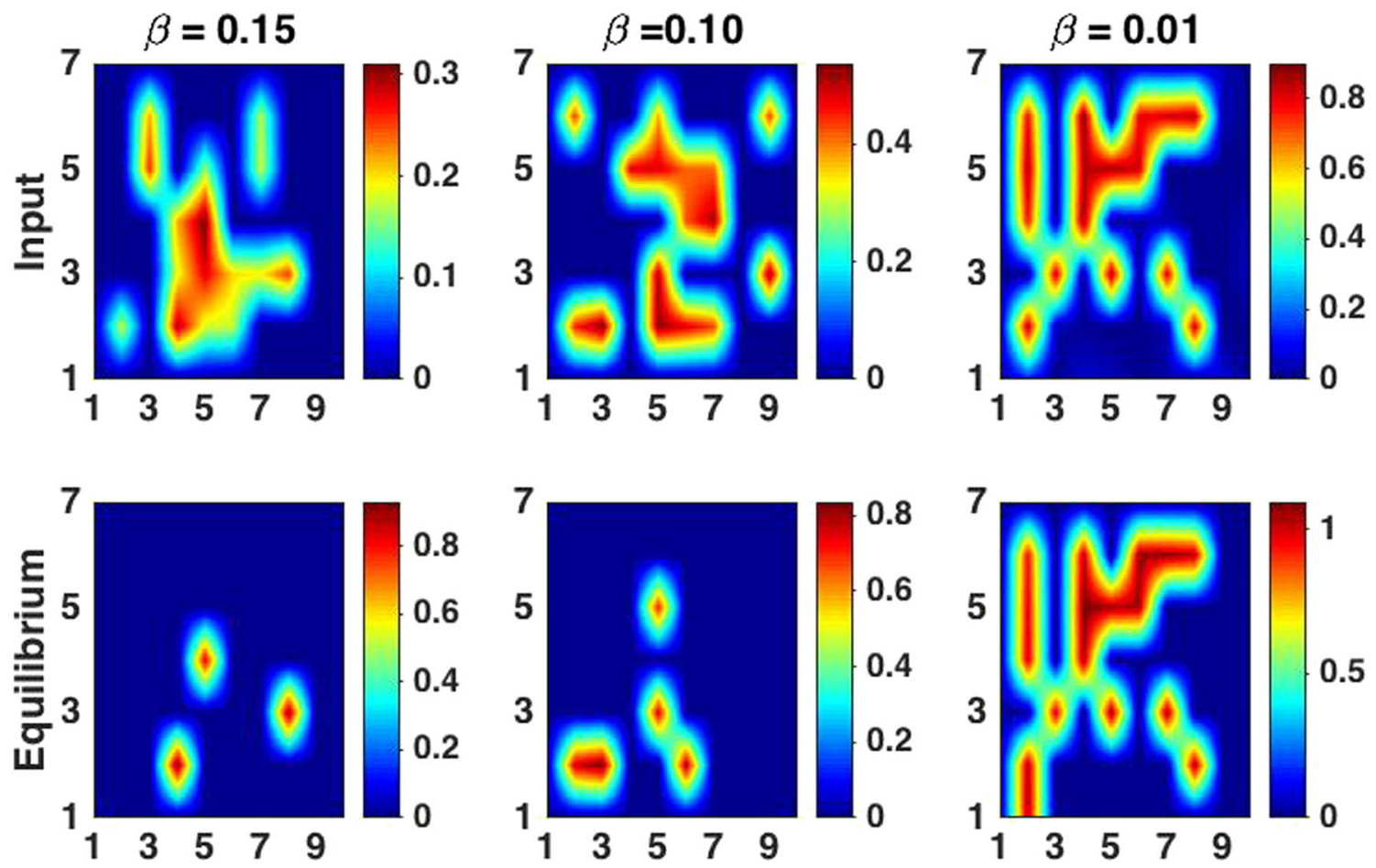

Fig. 2 Network dynamics for a large number of inputs (16) at different levels of $\beta$. Top panels show the initial activations and bottom panels show final activation on a $10 \times 7$ grid representing 70 nodes $(N=70)$

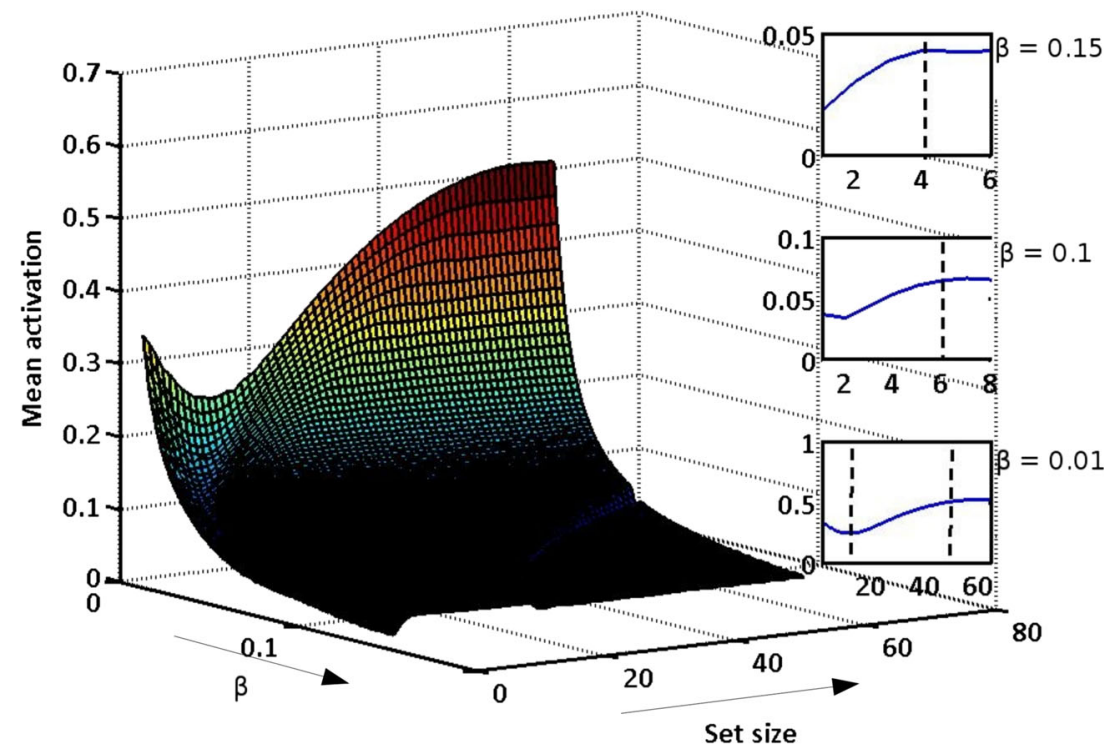

Fig. 3 Mean activation of the network of 70 nodes $\left(\bar{x}(n)=\sum x_{i} / N\right.$; i.e., the mean total activity per node) as a function of set size (the total number of external inputs to the network varied between 1 and 64) and $\beta$ (the inhibition parameter varied between 0.01 and 0.15 ). The plot was derived at fixed self-excitation $(\alpha=2.2)$ as an average of 100 simulations. For each simulation, the nodes chosen for particular set size were randomly chosen. The insets show the mean activation vs. set size plot for three particular $\beta$ values - low: 0.01 (bottom inset), medium: 0.1 (middle inset), and high: 0.15 (top inset). The insets show that mean activity of the network increases monotonically up to four items at high inhibition, up to around six items for medium inhibition and to higher ranges at low inhibition (reproduced from Sengupta et al., 2014). The dip in mean activation depicted for low $\beta$ signifies that the network output is not monotonically increasing with the number of inputs for smaller set sized and that the response is monotonic only for higher ranges of numerosity. Thus, the network is bad at subitizing at low inhibition 

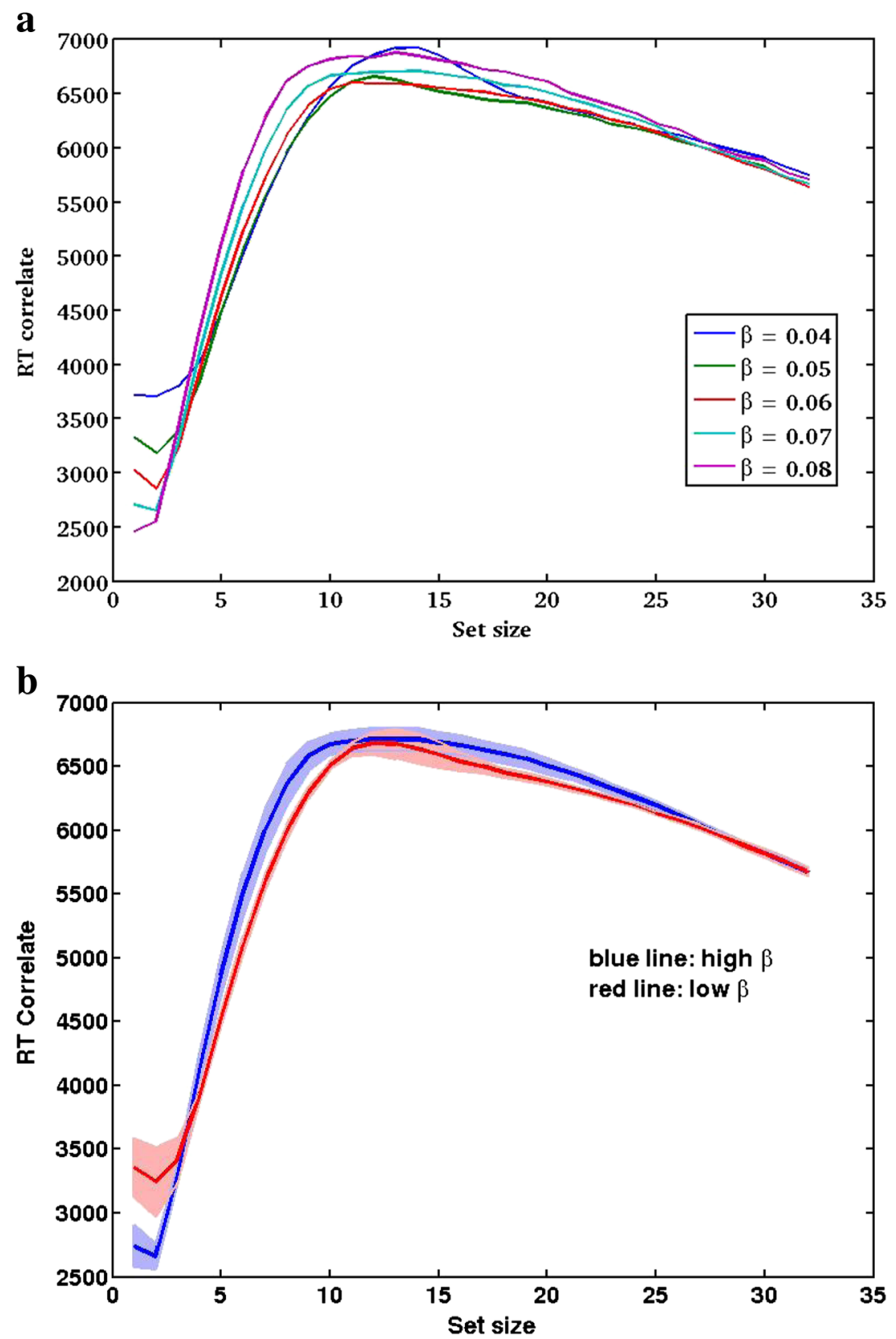

Fig. 4 Reaction time predictions from the model

Mind/Brain Sciences, University of Trento, Italy and nine additional participants were tested at the Center for Neural and Cognitive Sciences, University of Hyderabad, India. The participants included ten male and 11 female participants. Informed consent was obtained from all participants in accordance with the local ethical committee guidelines.

\section{Stimuli}

Stimuli were black dots $(20 \times 20$ pixels $)$ presented on a gray screen background within a virtual rectangle $(917 \times$ 683 pixels $)$ on a $(1280 \times 1024$ pixels $)$ monitor. There were two sets of numerosities: small $(1,2,3,4,5,6)$ and large $(24,26,28,30,32,34)$. The exact locations of the dots were randomized within the virtual rectangle on each trial.

\section{Design}

Participants were seated in a dimly lit room approximately $57 \mathrm{~cm}$ from a $45.2 \mathrm{~cm} \times 36.1 \mathrm{~cm}$ computer monitor. Following a short practice session (20 trials), participants began the experiment by pressing a keyboard button. After a brief delay (1 s), a set of dots was presented on the screen for 100 ms followed by a brief 50-ms flash. The flash was presented 


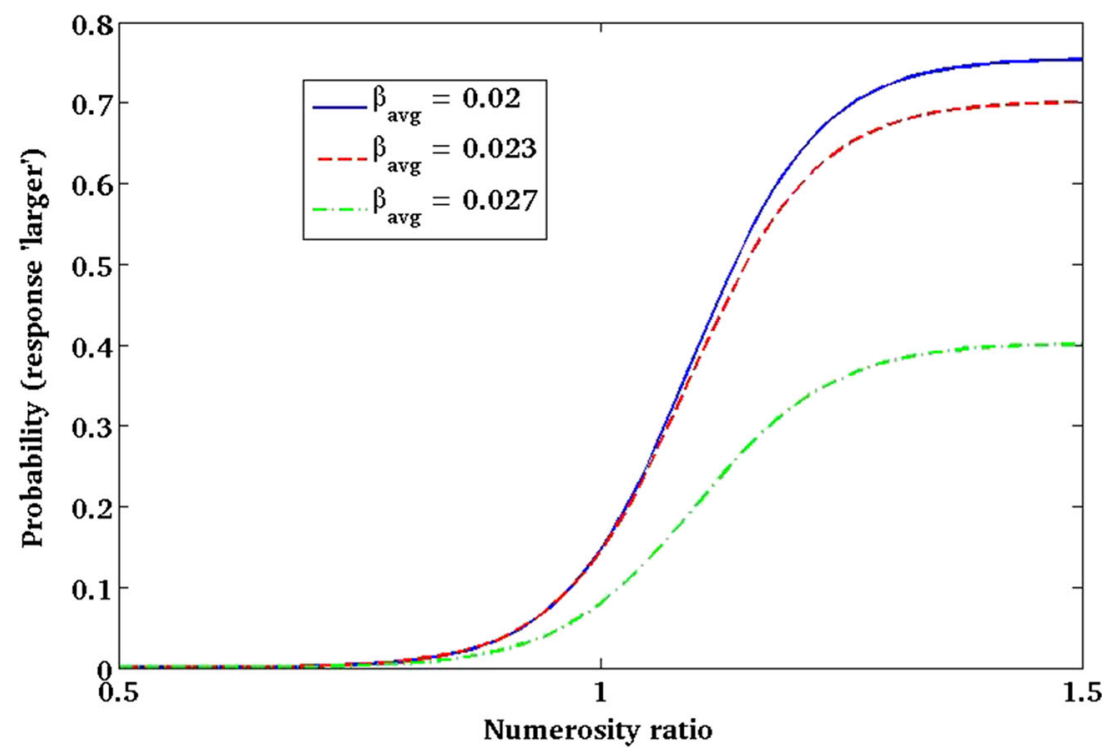

Fig. 5 Simulation of human numerosity comparison data by averaging the probability values at different average $\beta$. The plot clearly shows (i) the higher numerosities tend to be underestimated (the point of subjective equality or the $50 \%$ point corresponds to higher numerosity ratio), (ii) if we increase the average $\beta$, the point of subjective equality shifts right, pointing to higher underestimation

to reduce the visual persistence of the dots. After each trial, there was a 2-s interval prior to starting the next trial.

Participants were instructed to quickly and accurately verbally report the exact numerosity of dots presented on a computer screen. The schematics of the experiment are given in Fig. 7.

There were two types of trials: small numerosity trials or large numerosity trials. Within these two types of trials, the exact numerosity varied across trials, as described above. The experiment was designed so that there were sequences of trials in which the same type (small or large numerosity) was repeated. Each trial was associated with a Markov-like counter with probabilities of $-0.10,0.15,0.22,0.32,0.46$, 0.68 , or 1.00 . For instance, if the same kind of trial was shown five times in a row, then the switching probability (probability that the next trial would be from the other set of

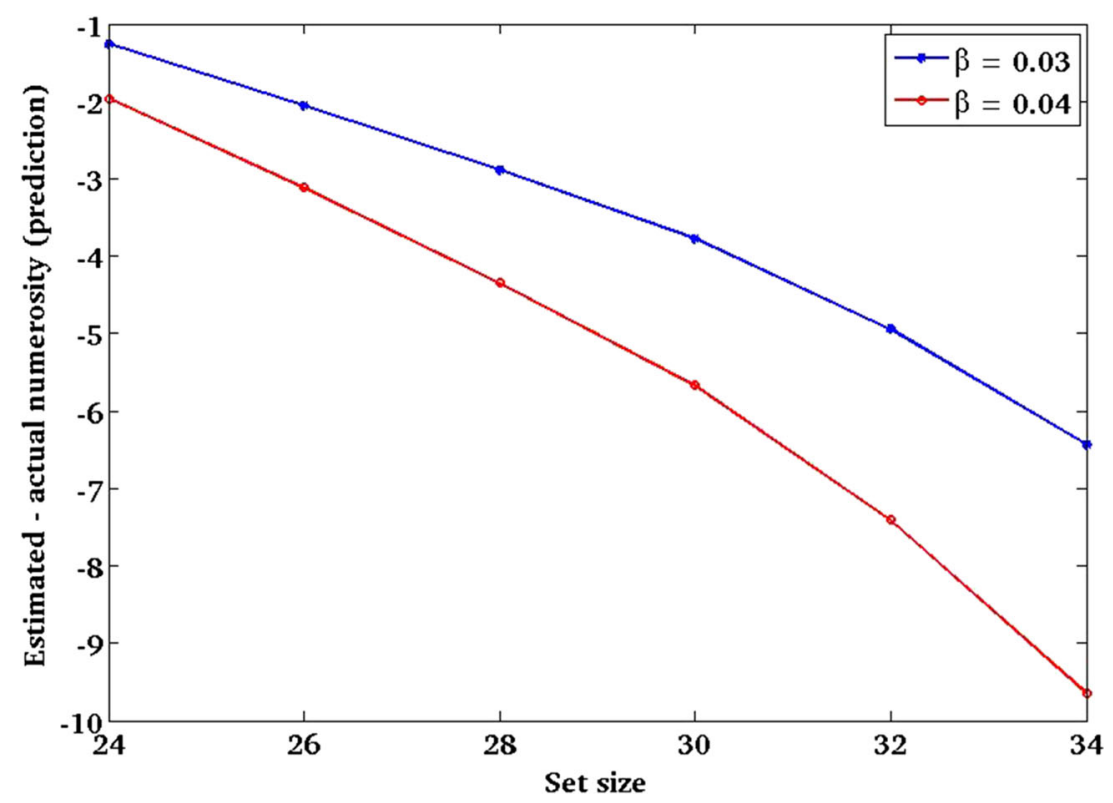

Fig. 6 Model prediction for estimation performance at different $\beta$ values. The prediction clearly shows that underestimation increases for increase in $\beta$. The numerosities shown here are the ones used in the empirical experiment 


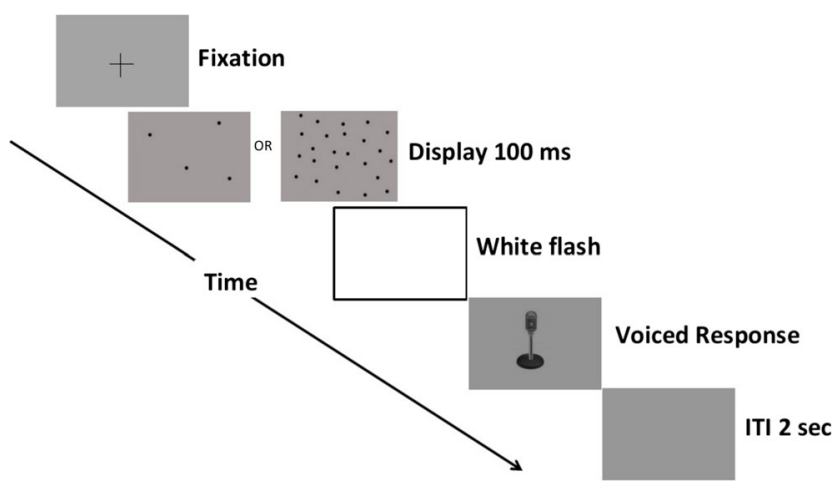

Fig. 7 Illustration of the structure of a trial. A display of black dots was presented for $100 \mathrm{~ms}$ and participants verbally reported the number of items as quickly and accurately as possible. The white flash was used as a mask to reduce the visual persistence of the dots in order to encourage participants to respond quickly rather than slowly counting each item individually from the after-image

numerosities) would become 0.46 . As a result, the number of trials in a sequence (of either large or small numerosity) ranged from 1 to 7 (average of 4). The total number of trials was 200 per subject and the total duration of the experiment was around $10 \mathrm{~min}$. Reaction time (RT is the voice onset time starting from when the flash terminates) was collected for each trial and the actual enumeration response was transcribed manually from the voiced response for each trial. Response error was computed by taking the difference between the reported and actual numerosities.

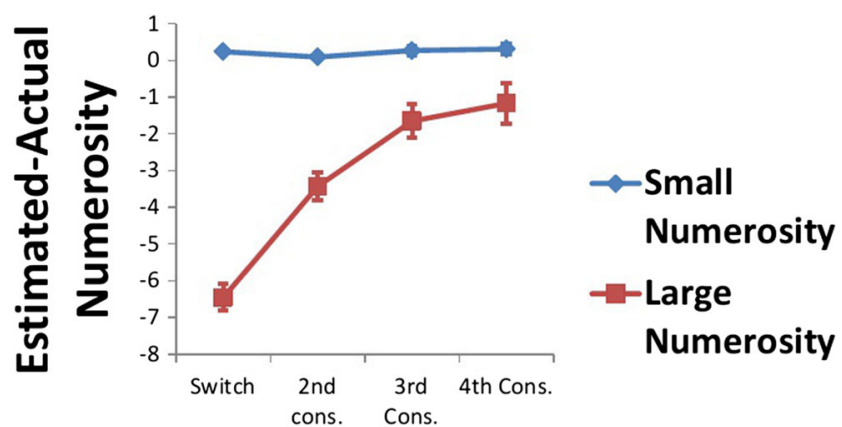

Condition

Fig. 9 The time course of underestimation effects of the switch, starting on first trial after the switch from small to large (red squares) or large to small (blue diamonds). The amount of underestimation is shown also for the 2nd, 3rd, and 4th consecutive numerosity trial of the same kind that continued after the switch

\section{Results}

\section{Accuracy and underestimation}

Consistent with previous findings in the literature, participants were highly accurate $(83 \%)$ and fast (mean RT $=717$ $\mathrm{ms}, \mathrm{SE}=19 \mathrm{~ms}$ ) for items presented within the subitizing range and considerably slower (mean $\mathrm{RT}=1156 \mathrm{~ms}$, $\mathrm{SE}=14 \mathrm{~ms}$ ) and less accurate for items presented within the estimation range. As predicted by the model, there was a strong tendency, within the large number range, to underestimate numerosity on switch trials compared to non-switch trials (Fig. 8). The specificity of this effect, occurring only

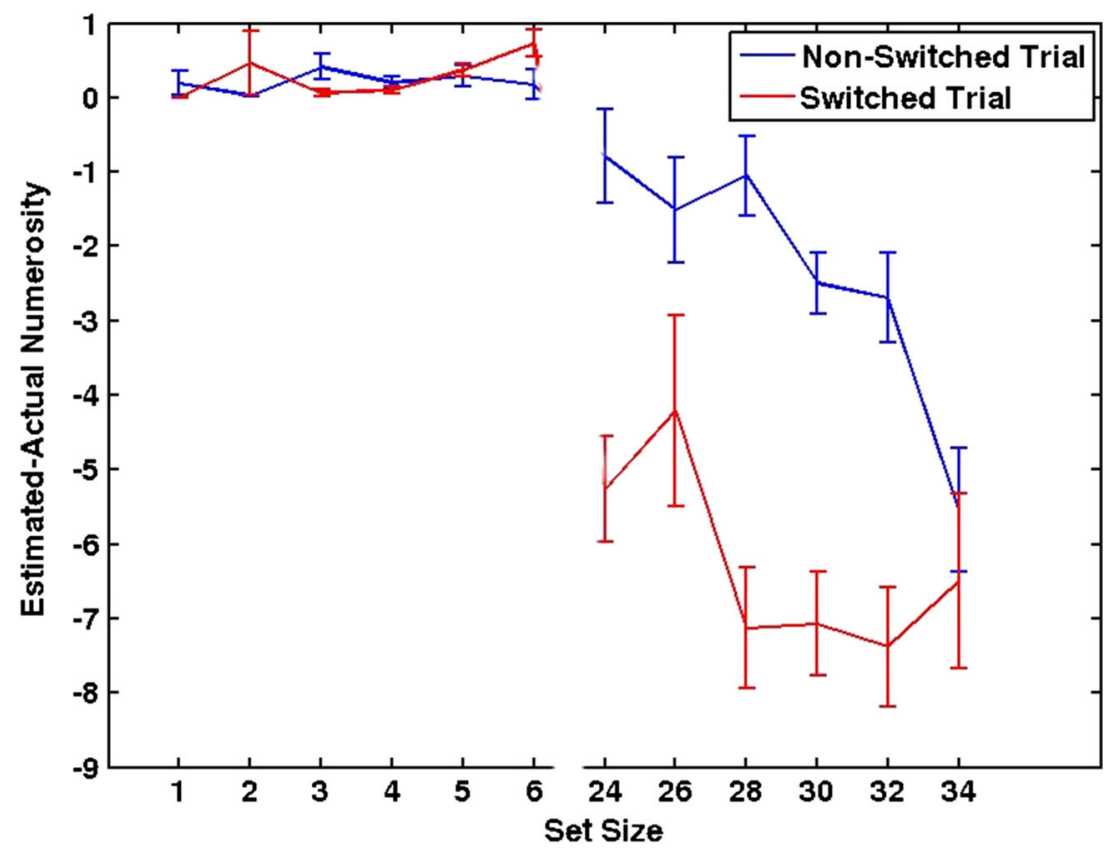

Fig. 8 Enumeration error between the reported numerosity by the participant (estimate) and the actual presented numerosity for different set sizes. Negative numbers indicate that the participants underestimated the numerosity. Error bars indicate standard error of the mean 


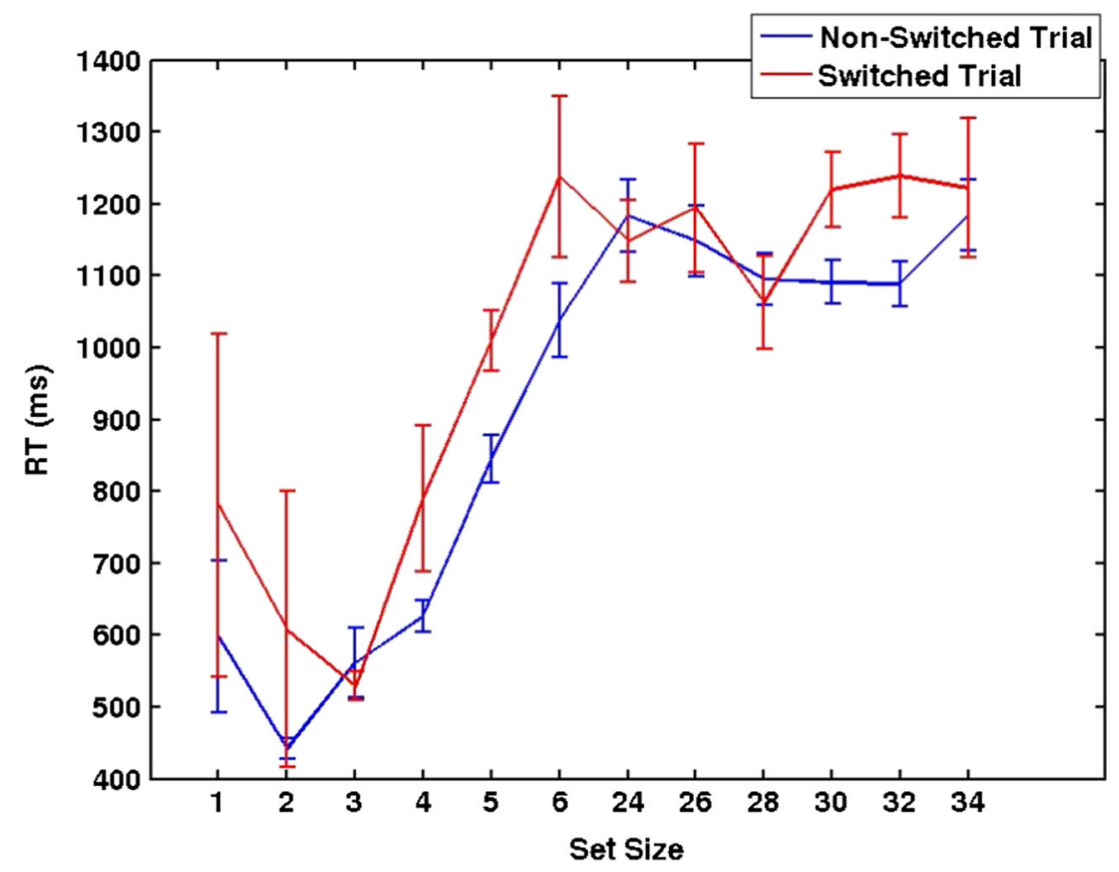

Fig. 10 Average reaction times for each numerosity plotted with error bars for standard/non-switched and switched trials. Non-switch trials refer to when a small numerosity trial followed a small numerosity trial or large numerosity trial followed a large numerosity trial (see "Model" section)

for the switch from small to big trials, is suggestive of a hysteresis effect from the small trials.

Further evidence for a hysteresis from small numerosity trials comes from the duration of the switch cost. As shown in Fig. 9, participants were increasingly more accurate with each trial following the switch from small to large (SL) numerosity. In other words, the initial switch cost for SL trials was reduced across trials and settled into a stable pattern of accuracy. In contrast, enumeration of small set sizes remained stable.

\section{Reaction time and switch cost}

As shown in Fig. 10, the reaction times for enumeration show standard features found by Kaufman et al. (1949), in

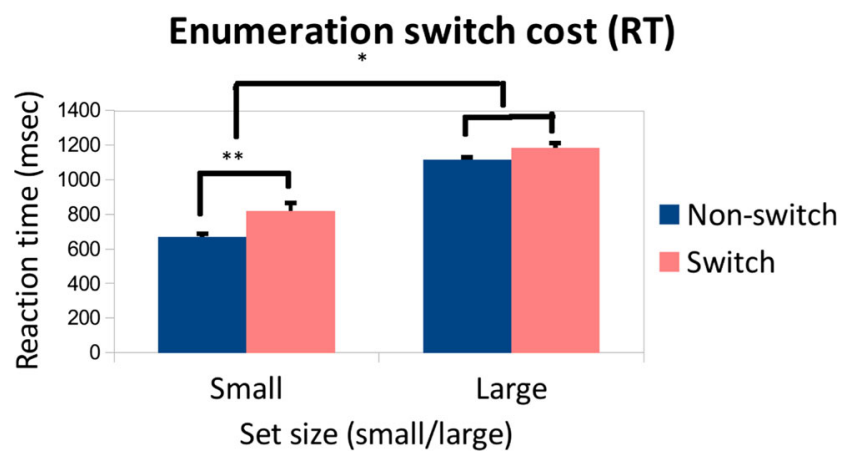

Fig. 11 Enumeration switch cost (RT) for reaction time for large and small numerosity trials that reaction time remained relatively fast for 1-4 items, then increased in a somewhat linear fashion (here, up to around six items). Interestingly, the average RT for six and 24 items was similar, suggesting that RT remained relatively constant as a function of set size after six items.

As predicted, there was a significant RT cost for switch trials when going from large to small numerosities. The main effects of set size, $F(1,20)=276.34, p<0.001$, and switch condition, $F(1,20)=16.75, p<0.001$ were confirmed in a two-factor repeated measures ANOVA. There was also a significant interaction between these two factors, $F(1,20)=4.06, p<0.05$. The results show a strong main effect for switch condition, $F(1,20)=276.34$, $p<0.001$ as well as a significant interaction between set

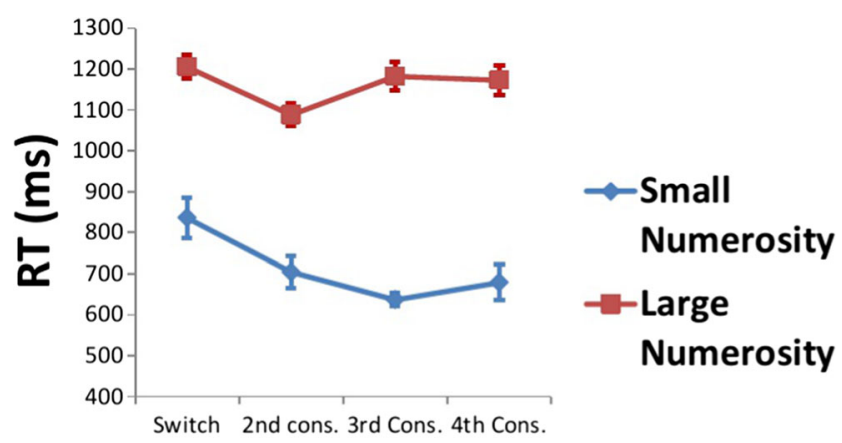

Condition

Fig. 12 Reaction time as a function of number of subsequent trials following the switch, for small and large numerosity trials 
size and switch condition, $F(1,20)=4.06, p<0.05$. The interaction appeared to be driven by the strong effect of switch only on small numerosity trials (Fig. 11). Given the interaction term, we performed a post hoc comparison of means (with Bonferroni correction) between switch categories within each set size class. We found an effect size of $\sim 150 \mathrm{~ms}, F(1,20)=13.48, p<0.001$ for large to small switch in terms of RT, whereas the cost for a small to large switch was not significant, $F(1,20)=3.44, p>0.05$. The results are shown in Fig. 11.

Also of interest was the duration of the switch cost. We separated the trials for small and large numerosity according to their distance from a corresponding switched trial, e.g., a small numerosity trial following a switched small numerosity trial (the last trial in the series L-S-S) would be called the 2 nd consecutive small numerosity trial, the second large numerosity trial following a switched large numerosity trial (the last trial in the series S-L-L-L) will be the third consecutive large numerosity trial, etc. As indicated in Fig. 12, reaction times decreased when small numerosities were repeated multiple times $(F(1,20)=$ 4.87, $p<0.05$ between switched $\mathrm{S}$ trial and the third consecutive S or Switch+4 trial), whereas no such difference was found between switch trials and switch +4 trials with large numerosities, $F(1,20)=0.36, p>$ 0.05 . The pattern of results after the switch is consistent with the network settling back into a stable state of low energy.

\section{Statistical permutation test}

The significance of this pattern of results was checked further with permutation tests. We randomized the trial orders 10,000 times for each participant and then performed the above analysis for each iteration. The test factor for us was the F-statistic for the switch vs. non switch trials for small and large numerosities, respectively. Thus we tested how many times out of 10,000 we would get a result greater than $F=16.75$ for small numerosity switches and how many times we get greater than $F=3.44$ for large numerosity switches. The probability for such an extreme result in case of small numerosities was calculated to be less than 0.001 and for large numerosities it was around 0.08 .

\section{RT switch cost and length of preceding incongruent trials}

One of the corollaries of our account is that for the large to small switch, the RT switch cost should be correlated positively with the number of large numerosity trials that precede the switch. On the other hand, we expect little effect of the length of small numerosity string that precedes a large numerosity trial on the reaction time. In the results, we can see that the large-to-small switch trial RTs increase linearlywith the number of preceding incongruent trials. However, no such effect is seen in RTs corresponding to small-to-large switch trials (see Fig. 13).

\section{Model predictions against empirical tests}

In order to check the model predictions against the empirical data, we considered two main predictions with regard to RTs. Firstly, mean RTs for numerosity judgments in the non-switch condition for set sizes 1-6 is predicted to be correlated with the mean RT estimate corresponding to a high inhibition value for the $\beta$ in the model. Secondly, mean RTs for numerosity estimation in the non-switch condition for large set sizes would be correlated with the RT estimates from the model operating with a low inhibition parameter value $\beta$, whereas in the switch condition, the RT values are expected to be correlated with high inhibition RT estimates for large numerosities and with low inhibition RT estimates for small numerosities. We first estimated a linear fit between the empirical values for the average RTs in nonswitch trials against the corresponding values obtained from the model.

$$
R T_{\text {non-switch }}(\text { empirical })=a * R T_{\text {non-switch }}(\text { model })+b
$$

There was a very good fit between the model and the data (adjusted $r^{2}=0.93$ at $95 \%$ confidence interval; see Fig. 14).

The coefficient values obtained were $a=1.097$ and $b=$ -1.267 .

We used the same coefficients to check the goodness of correlation between $R T_{\text {switch }}$ (empirical) and $R T_{\text {switch (model). Using the same coefficients } a \text { and } b \text {, we }}$ found a correlation coefficient of 0.9 at $95 \%$ confidence interval for the fit between model-derived and empirical RT on switch trials (see Fig. 15). The fits suffer significantly when we use very different $\beta$ choices from the ones mentioned here.

We have also compared the ideal observer performance with the median RT values obtained from the participants. The ideal observer RTs were calculated by using the curve fitting formula for the non-switch condition for the RT correlates calculated from the model. It is easy to see from Figs. 16 and 17 that the correlations obtained from curve fitting are not spurious, but are indicative of how well the model reflects actual human behavior.

One concern here might be that the correlations in Figs. 14 and 15 might be driven by a third underlying shared variable numerosity. However, we would like to point out that in the calculation of RT correlate (see Eq. 7 in the Appendix, and Sengupta et al. (2014)), the number of inputs or the numerosity does not figure as an explicit variable, 


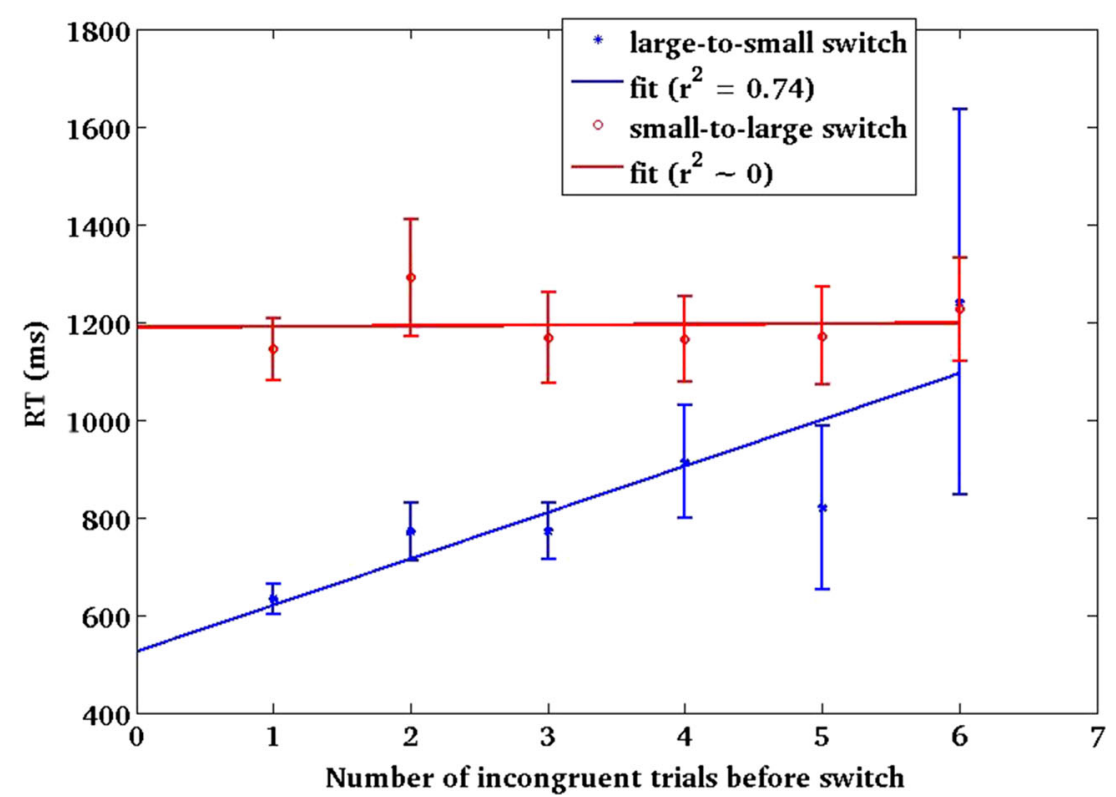

Fig. 13 Effect of number of intervening incongruent trials on switch cost for RT. $X$-axis shows how many large numerosity trials precede the small numerosity trial (for example, trial order for large-to-small switch: 1 refers to LS, 2 refers to LLS, 3 refers to LLLS and so on) or how many small trials precede the large numerosity trial (for example, trial order for small-to-large switch: 1 refers to SL, 2 refers to SSL, 3 refers to SSSL and so on). The large-to-small switch is shown in blue and the small-to-large switch is shown in red. The linear fit along with corresponding $r^{2}$ values are given in the figure

rather RT emerges as a calculation from the complexity of the network dynamics that drives it to steady state. Thus, the correlations observed here cannot be attributed solely to the shared variable of numerosity.

\section{Discussion}

In our previous work, we have shown how a single, flexible model of object individuation and number perception can account for several important phenomena in the domain of human numerical cognition (e.g., distance effect, Fechner's law) as well as human numerosity comparison and neuroimaging data (Knops et al., 2014). The current model is different from previous attempts at modeling visual number (Dehaene \& Changeux, 1993; Grossberg \& Repin, 2003; Stoianov \& Zorzi, 2012) because it did not assume the distinction between large and small numerosity a priori, but rather allowed the diverse number ranges to emerge through self-organization of the same network. In fact, the network effectively produces local-global dichotomy through network inhibition, in the sense that at higher inhibition the network can be seen as emulating focused (local) attention and at lower inhibition it shows characteristics of diffused (global) attention. An interesting and novel aspect of our approach is the modeling of reaction times based on an energy function. This formulation led to the prediction of specific asymmetric switch costs between small and large numerosities in terms of reaction times. The model output also led to the prediction of severe underestimation following a small-to-large enumeration switch. A hallmark of any successful computational model lies in its ability to offer testable predictions as well as explain known phenomena.

We reiterate here that the simulation experiments directly point us to the critical role $\beta$ plays in asymmetric impairments in switch conditions. These are depicted clearly in the parametric investigation reported in 3D plot in Fig. 3, RT changes with respect to switch in Fig. 4 as well as the underestimation effect as shown in Fig. 5. Now, there are two outcomes from these simulation results-one is the formulation of a testable prediction, and the other is the understanding of the putative role $\beta$ plays in these impairments. In the current work, we set out to test the prediction of the model in a behavioral enumeration task. We did find that for small numerosities there was a significant switch cost of around $150 \mathrm{~ms}$ for the transition from large numerosity estimation to small numerosity enumeration, whereas no significant switch cost was found for a small-to-large switch. The reaction time prediction from the model was highly correlated with the empirical data for both the non-switch and switch conditions in a linear fit (Figs. 14 and 15). Critically, the same parameters yielded good results for both numerical ranges and switch conditions. This formulation led to the prediction of severe underestimation following a small-large enumeration switch. The model's prediction of large underestimation following a small-to-large switch was also confirmed in error measurements. 


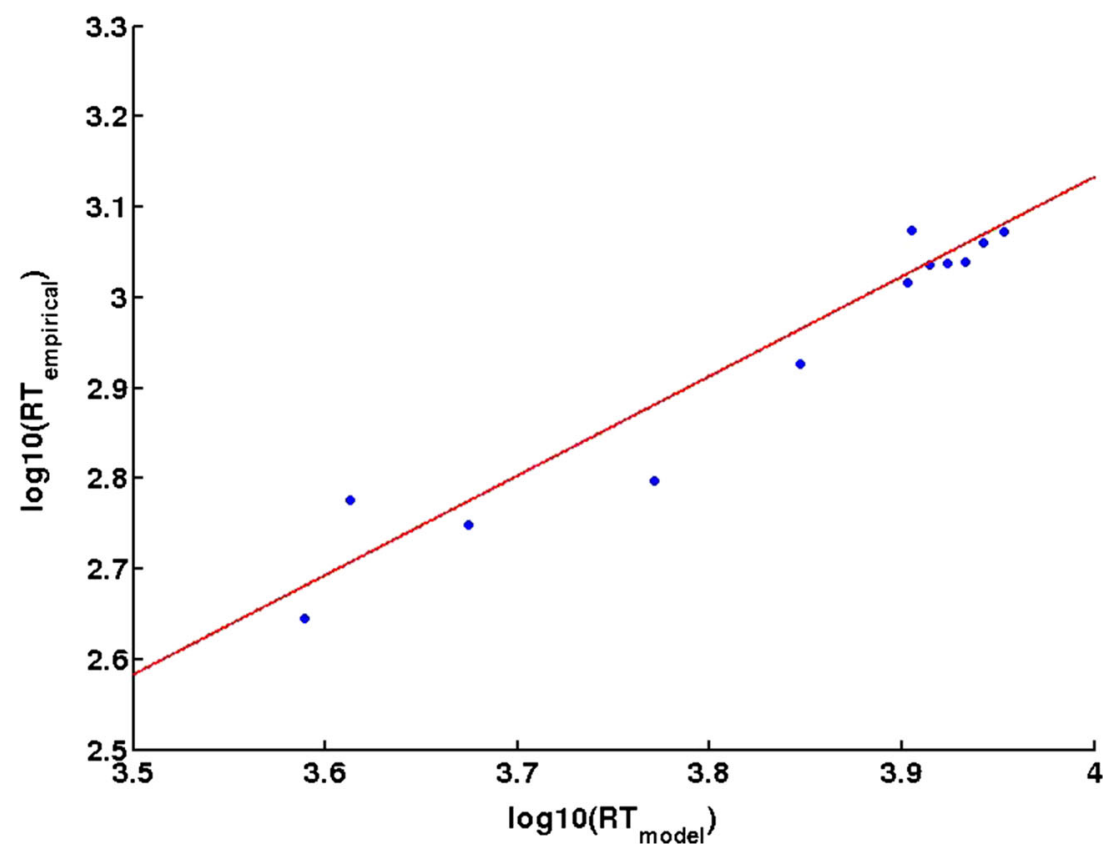

Fig. 14 The fit between model predicted RT values and empirically obtained RT values for non-switch trials $\left(R T_{\text {non-switch }}(\right.$ empirical $)=$ $a * R T_{\text {non-switch }}($ model $\left.)+b\right)$. Adjusted $r^{2}$ is 0.93 at $95 \%$ confidence interval

Asymmetric switch costs have been reported in the taskswitching literature (e.g., Gilbert and Shallice (2002)). In contrast, participants in the current study always performed the same task, with only the numerosity range changing from trial to trial. Moreover, there was a specific pattern of results, which differed for RT and error (underestimation) as a function of whether the switch was from large-tosmall or small-to-large, which does not map easily onto a general, non-specific task switching cost. Interestingly, Cicchini, Anobile, and Burr, (2014) have recently shown that in enumeration the previous trial's numerosity affects the current trial performance by up to $15 \%$ of the trial

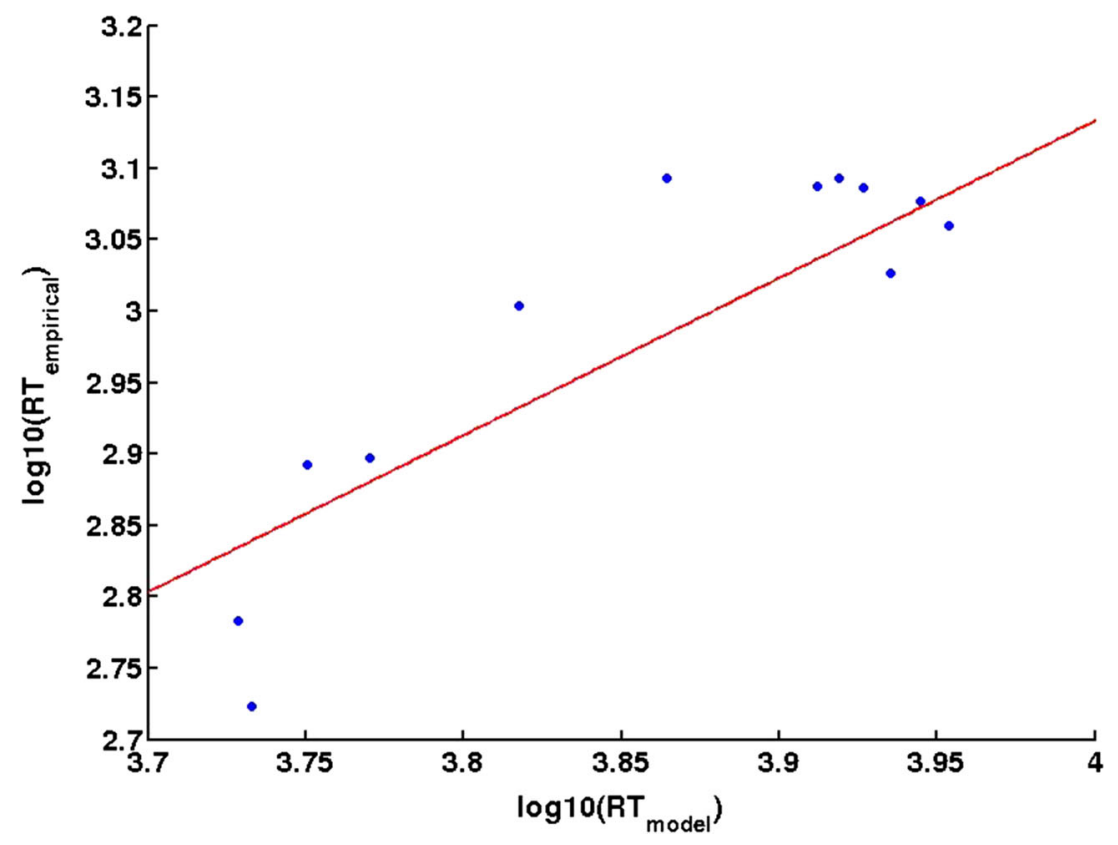

Fig. 15 A fit between model predicted RT values and empirically obtained RT values for switch trials using the same coefficients $a$ and $b$ obtained from the fit for non-switch trials 


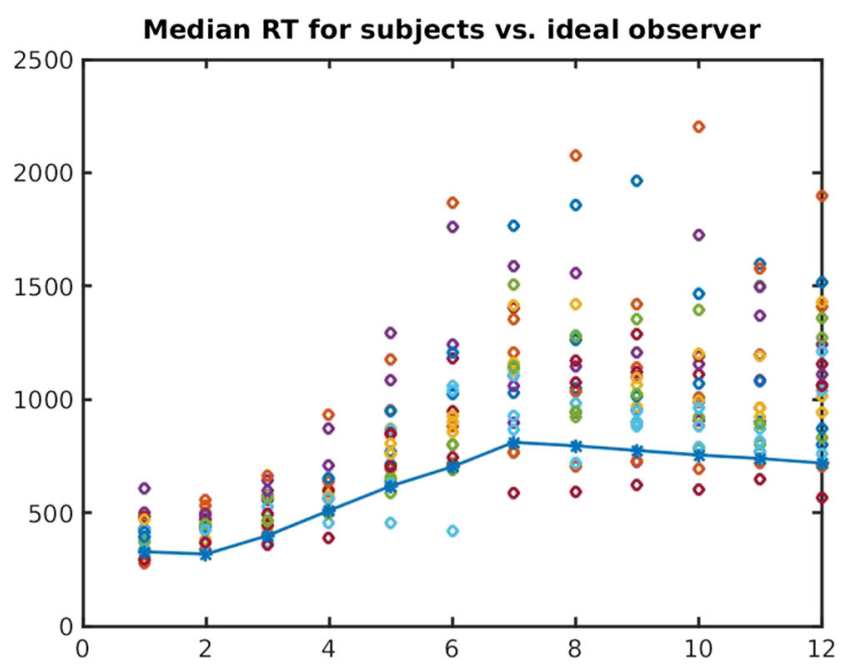

Fig. 16 The median RT value in ms for each numerosity for actual participants against an ideal observer (plotted with solid line) for the non-switch condition

numerosity value. Their experiment explicitly asks participants to respond to numerosity by mouse click on a linear scale (1-100). This motivates the prior-based strategies for the participants. Moreover, the study looked at the estimation ranges for their effect of priors. They offered an interesting Bayesian framework, which explained numerosity effects without resorting to the logarithmic number line common in literature. We do think some of our results for the asymmetric switch effects in both small-to-large and large-to-small numerosity switches need a more elaborate formulation. At this point, we do not rule out a Bayesian prior-based explanation for our results. In fact, the history effects may be due to resetting of network inhibition based on Bayesian priors in line with Cicchini et al. (2014).

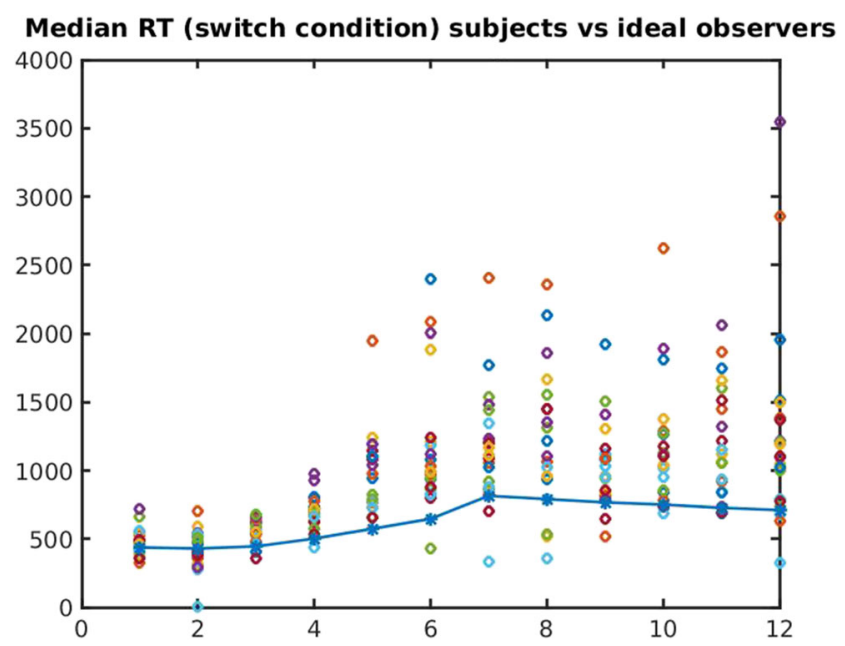

Fig. 17 The median RT value in ms for each numerosity for actual participants against an ideal observer (plotted with a solid line) for the switch condition
Another interesting aspect of the results is the fast recovery for RT in case of large-to-small switch (Fig. 12)) and slow recovery of underestimation accuracy following smallto-large switch (Fig. 9). We intend to investigate these in our future studies when we add a mechanism for automatic updating of $\beta$ to the model.

It might be possible that small and large numbers are processed by related yet distinct networks that share a common architecture with different inhibition parameters. This less parsimonious account does lead to some difficulties in explaining the current findings. The reaction time switch cost from large to small numbers can be explained in the account with two networks, but then we have to assume different interaction weights for the small-to-large switch, which gives almost no switch cost in terms of RT. On the other hand, the characteristic underestimation in the case of a small-to-large switch does not translate into an overestimation for a large-to-small switch (although the accuracy suffers). It is also difficult to explain the duration of switch costs from trial to trial (as seen in Figs. 9 and 12) in the two-network account. A single, flexible mechanism is more parsimonious and able to account for all the observed effects within the same network. Moreover, there is some preliminary neuroimaging evidence for a single, flexible network (Roggeman et al., 2010; Knops et al., 2014).

One alternative explanation for our results would be a general hysteresis. However, such a general effect would not account for the specific pattern of effects found here. One advantage of the model proposed here is that it accounts for a diverse range of effects (Sengupta et al., 2014; Knops et al., 2014) and provides a mechanistic explanation of why hysteresis might occur.

Overall, the ability of a single, flexible model to account for human performance in both small and larger number ranges has implications towards the debate regarding mechanisms of subitizing and estimation (Whalen et al., 1999; Melcher \& Piazza, 2011; Revkin et al., 2008; Dehaene \& Changeux, 1993). One possibility, suggested by recent work looking at the role of attention in numerical judgments Burr et al. (2010), is that the use of focused attention in order to individuate a small number of items acts to increase the inhibition in the network. This idea is consistent with attention models, such as the biased competition model, which account for the ability of neurons to respond to an attended stimulus in the presence of distractors. Based on previous neuroimaging and neurophysiological studies, neurons representing an attentional priority (saliency) map in posterior parietal cortex (Roggeman et al., 2010; Knops et al., 2014) might act in a similar way to the recurrent on-center off-surround network model used here. In other words, the remarkable overlap among studies of attention, object individuation and working memory, number, and eye movements in the LIP area (Piazza et al., 2007) may not 
simply be a coincidence but may rather implicate a shared, flexible attention priority map for representing the presence of important objects in the visual environment.

Acknowledgments This work was supported by a European Research Council Starting Grant (StG-20111124) to D.M. and by the India-Trento Programme for Advanced Research.

\section{Appendix: Reaction time from network dynamics}

In our previous work (Sengupta et al., 2014), we took a novel approach towards characterizing network stability in terms of a Hamiltonian function. It had the advantage of explaining the analytic solution space of the network in more natural terms, as well as deriving constraint equations that were not visible from network analysis alone, but could be confirmed through simulations (see Appendix of Sengupta et al. (2014) for detailed derivation and further explanations).

$H=\sum_{i} H_{i} \propto-\sum_{i} \int\left(1-\alpha\left(\frac{F\left(x_{i}\right)}{x_{i}}\right)^{2}\right) \dot{x}_{i}^{2} d t$

$\dot{x}_{i}=\frac{d x_{i}}{d t}$ and $H_{i}$ is the energy for a particular node $i$.

The driving argument behind the formulation of reaction time for enumeration was that the reaction time should correlate with maximum allowed fluctuation of energy for the network. Thus we postulated

$R T \sim \sum_{i} \int\left(1-\alpha\left(\frac{F\left(x_{i}\right)}{x_{i}}\right)^{2}\right) \dot{x}_{i}^{2} d t$

\section{References}

Ariely, D. (2001). Seeing sets: representation by statistical properties. Psychological Science, 12, 157-162.

Awh, E., \& Jonides, J. (2001). Overlapping mechanisms of attention and spatial working memory. Trends in Cognitive Science, 5, 119-126.

Burr, D., \& Ross, J. (2008). Visual sense of numbers. Current Biology, $18,425-428$.

Burr, D., Turi, M., \& Anobile, G. (2010). Subitizing but not estimation of numerosity requires attentional resources. Journal of Vision, 10, $1-10$.

Choo, H., \& Franconeri, S.L. (2014). Enumeration of small collections violates Weber's law. Psychon Bull Rev, 21(1), 93-99.

Cicchini, G.M., Anobile, G., \& Burr, D.C. (2014). Compressive mapping of number to space reflects dynamic encoding mechanisms, not static logarithmic transform. Proceedings of the National Academy of Sciences of the United States of America, 111(21), 7867-7872.

Dehaene, S., \& Changeux, J.P. (1993). Development of elementary numerical abilities: a neuronal model. Journl of Cognitive Neuroscience, 5, 390-407.

Feigenson, L., Dehaene, S., \& Spelke, E.S. (2004). Core systems of number. Trends in Cognitive Sciences, 8, 307-314.
Franconeri, S.L., Alvarez, G.A., \& Cavanagh, P. (2013). Flexible cognitive resources: competitive content maps for attention and memory. Trends in Cognitive Sciences, 17, 134-141.

Gallistel, C.R., \& Gelman, R. (1992). Preverbal and verbal counting and computation. Cognition, 44, 43-74.

Gilbert, S.J., \& Shallice, T. (2002). Task switching: a PDP model. Cogn Psychol, 44, 297-337.

Gottlieb, J., \& Goldberg, M.E. (1999). Activity of neurons in the lateral intraparietal area of the monkey during an antisaccade task. Nature Neuroscience, 2, 906-912.

Grossberg, S., \& Repin, D.V. (2003). A neural model of how the brain represents and compares multi-digit numbers: spatial and categorical processes. Neural Networks, 16, 1107-1140.

Halberda, J., \& Feigenson, L. (2008). Set representations required for the acquisition of the "natural number" concept. Behavioral and Brain Sciences, 31, 655-656.

Kaufman, E.L., Lord, M.W., Reese, T., \& Volkmann, J. (1949). The discrimination of visual number. American Journal of Psychology, $62,498-525$.

Kawasaki, M., Watanabe, M., Okuda, J., Sakagami, M., \& Aihara, K. (2008). Human posterior parietal cortex maintains color, shape and motion in visual short-term memory. Brain Research, 1213, 91-97.

Knops, A., Piazza, M., Sengupta, R., Eger, E., \& Melcher, D. (2014). A shared, flexible neural map architecture reflects capacity limits in both visual short term memory and enumeration. Journal of Neuroscience, 34(30), 9857-9866.

Melcher, D., \& Piazza, M. (2011). The role of attentional priority and saliency in determining capacity limits in enumeration and visual working memory. PLoS One, 6, 1-11.

Piazza, M., Fumarola, A., Chinello, A., \& Melcher, D. (2011). Subitizing reflects visuo-spatial object individuation capacity. Cognition, $121,147-53$.

Piazza, M., Izard, V., Pinel, P., Le Bihan, D., \& Dehaene, S. (2004). Tuning curves for approximate numerosity in the human intraparietal sulcus. Neuron, 44, 547-555.

Piazza, M., Pinel, P., Le Bihan, D., \& Dehaene, S. (2007). A magnitude code common to numerosities and number symbols in human intraparietal cortex. Neuron, 53, 293-305.

Revkin, S.K., Piazza, M., Izard, V., Cohen, L., \& Dehaene, S. (2008). Does subitizing reflect numerical estimation? Psychological Science, 19, 607-14.

Reynolds, J.H., \& Heeger, D.J. (2009). The normalization model of attention. Neuron, 61(2), 168-185.

Roggeman, C., Fias, W., \& Verguts, T. (2010). Salience maps in parietal cortex: imaging and computational modeling. NeuroImage, $52,1005-1014$.

Roitman, J.D., Brannon, E.M., \& Platt, M.L. (2007). Monotonic coding of numerosity in macaque lateral intraparietal area. PLoS Biology, 5, 1673-1682.

Sengupta, R., Surampudi, B.R., \& Melcher, D. (2014). A visual sense of number emerges from the dynamics of a recurrent on-center off-surround neural network. Brain Research, 1582, 114-124.

Stoianov, I., \& Zorzi, M. (2012). Emergence of a 'visual number sense' in hierarchical generative models. Nature Neuroscience, 15, 194-196.

Todd, J.J., \& Marois, R. (2004). Capacity limit of visual short-term memory in human posterior parietal cortex. Nature, 428, 751-754.

Todd, J.J., \& Marois, R. (2005). Posterior parietal cortex activity predicts individual differences in visual short-term memory capacity. Cognitive, Affective, \& Behavioral Neuroscience, 5, 144-55.

Trick, L.M., \& Pylyshyn, Z.W. (1994). Why are small and large numbers enumerated differently? A limited-capacity preattentive stage in vision. Psychological Review, 101, 80-102.

Whalen, J., Gallistel, C.R., \& Gelman, R. (1999). Nonverbal counting in humans: the psychophysics of number representation. Psychological Science, 10, 130-137. 